Classification

Physics Abstracts

$76.60 \mathrm{E}-61.65$

\title{
Dynamic disorder in stable and metastable thiophene. I. N.M.R. lineshape study and structurally based interpretations
}

\author{
D. André, C. Bessada, A. H. Fuchs, B. Rousseau and H. Szwarc \\ Laboratoire de Chimie-Physique des Matériaux Amorphes, U.A. 1104 du C.N.R.S., Bât. 490, Université \\ Paris-Sud, 91405 Orsay, France
}

(Reçu le 6 août 1987, accepté le 19 octobre 1987)

\begin{abstract}
Résumé. - Nous avons effectué une étude de R.M.N. à raies larges du thiophène cristallin à l'aide d'un spectromètre à haut champ et transformée de Fourier. Nous avons observé les séquences stable et métastable ainsi que les transitions de phases, en très bon accord avec nos précédents travaux de calorimétrie [1]. Nous avons calculé les seconds moments théoriques à l'aide des données radiocristallographiques de André et al. [2]. Nous avons montré que les nombreuses configurations locales différentes compatibles avec les modèles structuraux conduisaient à une très large distribution des valeurs possibles de la contribution intermoléculaire au second moment en réseau rigide. Dans la gamme de température accessible $(120$ à $240 \mathrm{~K})$, et probablement à plus basse température, toutes les phases solides sont le siège d'un désordre orientationnel dynamique dû à la réorientation des molécules dans leurs plans. La fréquence du mouvement reste de l'ordre de grandeur de celle du champ dipolaire local dans toute la gamme expérimentale de température, ce qui montre l'existence d'une large distribution de temps de corrélation du mouvement. Ce phénomène est sans doute caractéristique des composés moléculaires formant des cristaux vitreux.
\end{abstract}

Abstract. - A proton wide-line N.M.R. study of crystalline thiophene has been undertaken using a high sensitivity F.T. spectrometer. Both stable and metastable phase sequences have been observed and phase transitions have been detected in very good agreement with our previous calorimetric studies [1]. Theoretical second moments have been calculated using the X-ray scattering data of André et al. [2]. Given the structural models, we have shown that the many different allowed configurations lead to a wide distribution of theoretical intermolecular second moments, suggesting very different local steric hindrances. All the known crystalline phases, from crystallization temperature down to $120 \mathrm{~K}$ and presumably lower, are orientationally disordered. The nature of this disorder was proven to be the in-plane molecular reorientation. The frequency of the motion is commensurate with the local field in a very wide range of temperatures indicating a wide distribution of correlation times. We suggest that this could be a signature for glassy crystalline-forming materials.

\section{Introduction.}

Crystalline thiophene has long been the object of misinterpretations until calorimetric measurements [3] revealed the existence of two parallel sequences of phases and pointed out the appropriate thermal treatment to get them. The phase diagram at atmospheric pressure is shown in table $\mathrm{I}$.

$\mathrm{X}$-ray scattering experiments have been performed in phases I and II as well as in the metastable $\mathrm{II}_{1}$ and $\mathrm{II}_{2}$ phases $[2,4]$. The best molecular packing for phase I corresponds to space group Cmca with 20 equiprobable molecular orientations, as shown in figure 1 , and 4 molecules per unit cell. Phases II, $\mathrm{II}_{1}$ and $\mathrm{II}_{2}$ are incommensurate. They correspond to superstructures of the orthorhombic phase I lattice. The structure of phase III has been investigated at high pressure [2], it is best described by the space group Pnma with 10 molecular orientations $(Z=2)$.

Finally, a further low temperature calorimetric work [1b] showed the occurrence of a glassy crystalline phase in both sequences (see Tab. I), thus providing the first example of a new kind of disordered materials : incommensurate glassy crystals. 
Table I. - Phase transitions in the two phase sequences of crystalline thiophene (from [4]). The arrow indicates the irreversible transformation $\mathrm{II}^{\prime}$ to $\mathrm{III}$ that occurs from the metastable sequence to the stable one.

\begin{tabular}{|c|c|c|c|c|c|c|c|c|c|}
\hline $\mathrm{T} / \mathrm{K}$ & \multicolumn{2}{|r|}{41.7} & 112.35 & 138.5 & \multicolumn{2}{|l|}{170.70} & 175.03 & \multicolumn{2}{|c|}{235.03} \\
\hline $\begin{array}{c}\text { Phases } \\
\text { (stable sequence) }\end{array}$ & $V_{g}$ & V & IV & III & $\uparrow$ & II & & I & liq. \\
\hline $\begin{array}{c}\text { Phases } \\
\text { (metastable sequence) }\end{array}$ & $\mathrm{II}_{2 \mathrm{~g}}$ & $\mathrm{II}_{2}$ & $\mathrm{II}_{1}$ & $\mathrm{II}^{\prime}$ & & II & & I & liq. \\
\hline$T / K$ & & 36.9 & 90.76 & 139.2 & 170.70 & & 175.03 & & \\
\hline
\end{tabular}

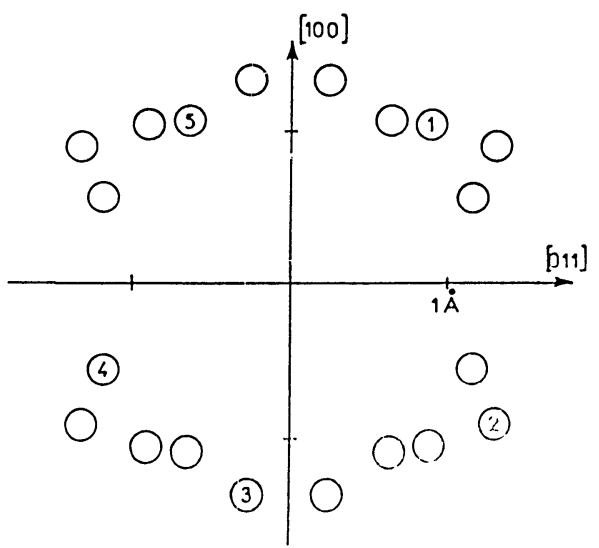

Fig. 1. - Thiophene, phase I : projections of the sulphur atoms of the 20 thiophene molecular orientations on a plane defined by directions [100] and [011] (from [2]).

Given this important experimental background, we have found it useful to reinvestigate the dynamics of molecular motions which, for reasons stated above, have been the matter of some confusion [5, $6]$. The relative simplicity of the molecule, a small and rigid one, could hopefully provide another insight on the relation between local dynamic disorder and glass transition.

\section{Experimental.}

Thiophene of $>99 \%$ purity was purchased from Janssen and thoroughly purified. Samples have been distilled twice under nitrogen atmosphere with a $300 \mathrm{~mm}$ spinning band column, dried on molecular sieve and sealed under vacuum. Its final purity is not exactly known, however a high resolution N.M.R. liquid analysis showed no sign of remaining benzene which is the main impurity.

Proton absorption lines have been obtained with a Fourier transform (FT) AM400 Bruker $400 \mathrm{MHz}$ spectrometer. Short pulses of $2 \mu \mathrm{s}$ (corresponding to an angle of $13^{\circ}$ ) were used in order to avoid signal distorsion due to power inhomogeneity, the record- ing frequency width being as large as $160 \mathrm{kHz}$ at low temperature. 32 F.I.D. (free induction decay) were accumulated at each temperature giving rise to a $S / N$ ratio of about 50. The F.I.D. transformed into an absorption line of approximately $50 \mathrm{~Hz} /$ point. The overall recovery time of the probe and receiver was $6 \mu \mathrm{s}$ as compared to $T_{2}$ values of $40 \mu \mathrm{s}$ or more, so that no more than $15 \%$ of the signal, obtained with quadrature detection, was lost.

The lowest temperature that could be reached with the Bruker probe and cooling facility was unhappily $110 \mathrm{~K}$.

\section{Results.}

3.1 LINE-WIDTH AND SECOND MOMENTS. - The proton line widths as a function of temperature are shown in figure 2 for both phase sequences. We proceeded as follows : after crystallization of the sample, temperature was lowered gradually down to $175 \mathrm{~K}$. The sample was then cooled rapidly to $110 \mathrm{~K}$ in order to avoid transformation into the stable phase III. We then reheated gradually while recording the absorption lines in the metastable sequence every $5 \mathrm{~K}$. At roughly $165 \mathrm{~K}$, the sample transformed irreversibly into the more stable phase III. After a 30 min annealing at $165 \mathrm{~K}$ we cooled down the sample gradually while recording the stable sequence absorption lines. In both sequences, we obtained a good reproducibility between the spectra recorded after different heat treatments.

As sketched in figure 2, some of the known phase transitions can be detected, the observed transition temperatures being in very good agreement with our previous calorimetric works [1]. The IV to III, $\mathrm{II}_{2}$ to $\mathrm{II}_{1}$ and $\mathrm{II}$ to $\mathrm{I}$ transformations only give rise to a change of slope. This means that both phases involved in each transition are very close to each other from the point of view of structure and dynamic behaviour. This agrees with what is known from X-ray scattering. The II to III transition is a sharp-one and is known to be first order from volumetric [2] and calorimetric [1b] measurements. 


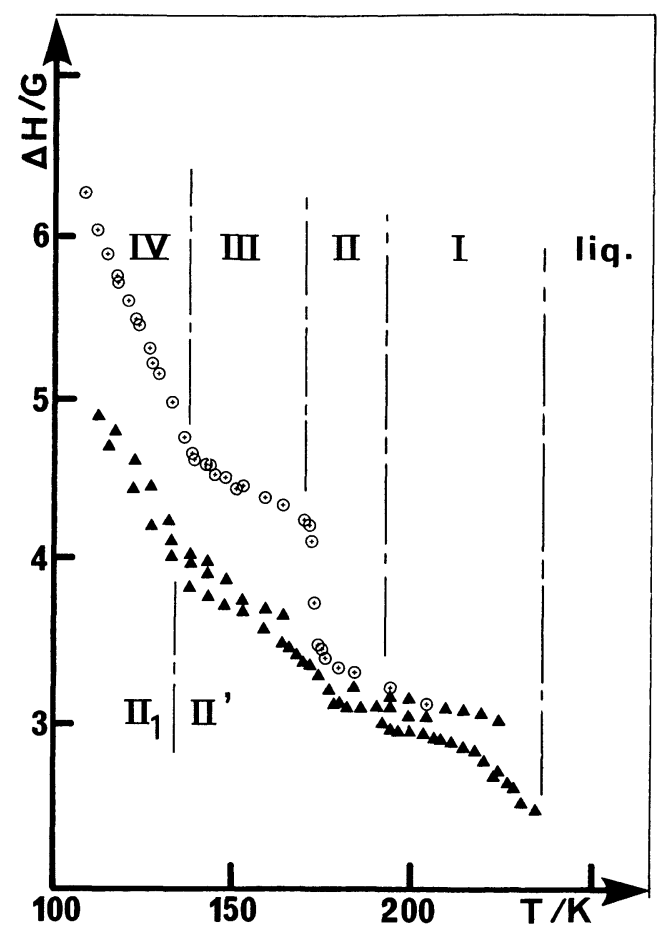

Fig. 2. - Line widths of the proton NMR absorption lines as a function of temperature : $\oplus$ stable sequence ; $\Delta$ metastable sequence.

The close similarity between the dynamic behaviour of the two phase sequences appears at a glance from figure 2 . All phases seem to be orientationally disordered and the ways motions slow down when lowering temperature are parallel, the more stable phases being only more « compact " than the metastable ones. This will be confirmed later on.

The lineshapes for all the observed phases are shown in figure 3 . The lines are homogeneous except those from the $\mathrm{II}_{1}$ and IV phases. We have tried different heat treatments without being able to get

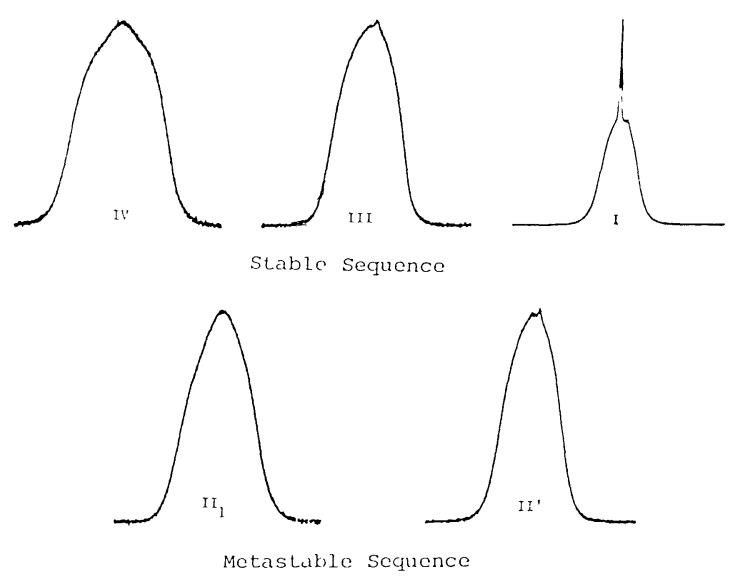

Fig. 3. - Lineshapes observed in different phases. rid of these inhomogeneities. This could be due to the incommensurate character of the phases involved. As information about the dynamic nature of incommensurability in those phases (from neutron scattering for instance) is lacking, we cannot draw any conclusion from this for the time being.

The approach to melting of phase $I$ is shown in figure 4. As can be seen, the onset of melting is characterized by a fine peak superimposed on the large phase I line. The width of the thin line is of the order of $500 \mathrm{~Hz}$ and is approximately constant throughout the premelting range. At $240 \mathrm{~K}$ the high resolution liquid spectrum is obtained. We have checked the absence of a vertical temperature gradient larger than $0.5 \mathrm{~K}$ by placing thermocouples at both ends of the sample inside the tube. No contact can occur between the spinning sample and the r.f. coil thus avoiding local thermal perturbation. Everything occurs as if a gradually larger part of the molecules in the sample was undergoing fast selfdiffusion at a rate commensurate with the local dipolar field. Fast self-diffusion is known to occur near melting in disordered molecular crystals [9]. It was usually thought of as being experienced by all

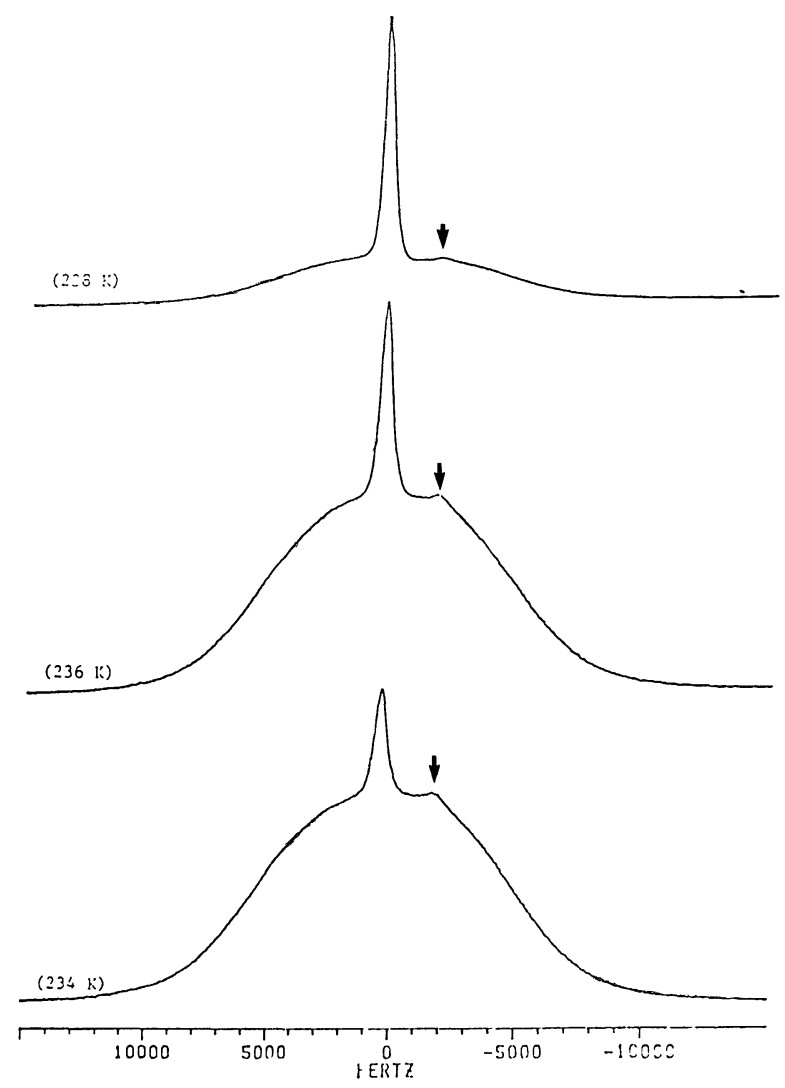

Fig. 4. - Evolution of the lineshape in phase I near melting. The melting point is $240 \mathrm{~K}$. The little peak indicated by an arrow is an artefact. It is presumably due to a snap of glue somewhere in our ${ }^{13} \mathrm{C} /{ }^{1} \mathrm{H}$ probe. It appears with the same intensity in the absence of sample. 
the molecules in the sample. The fast diffusing molecules could presumably lie at grain boundaries, near point defects, dislocations or impurities. This kind of « heterophase " premelting has been theoretically predicted by Bartis [10].

Figure 5 shows the calculated second moments of the recorded absorption lines. The same conclusions as for figure 2 can be drawn here except that the data are somewhat more scattered due to phase and baseline corrections that have been performed before computing the second moments (a baseline correction affects the wings of the line thus introduces an uncertainty on the second moment which has been experimentally estimated to be roughly $10 \%)$. In the next section these results are analysed in terms of structurally-based calculations.

3.2 THEORETICAL SECOND MOMENT CALCULATIONS. - Proton second moments have been

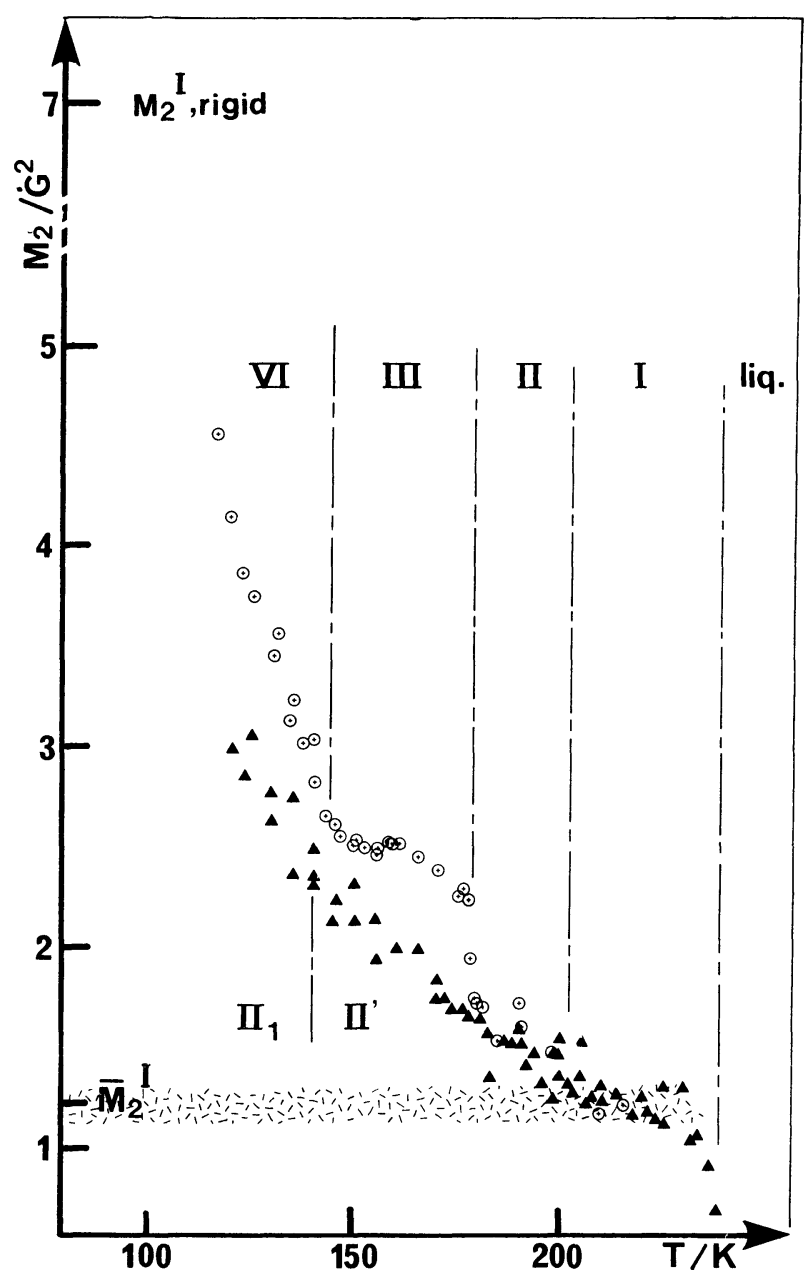

Fig. 5. - Calculated second moments of the recorded absorption lines : $\oplus$ stable sequence ; $\boldsymbol{\Delta}$ metastable se-

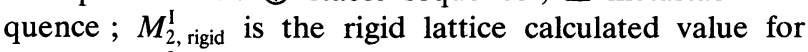
phase $I ; M_{2}^{1}$ is the calculated value assuming an in-plane molecular reorientation. calculated using the well known Van Vleck formula (7) :

$$
\begin{aligned}
M_{2}=(3 / 4) \gamma^{4} \hbar^{2} I(I & +1) N^{-1} \times \\
& \times \sum_{j>k}\left(1-3 \cos ^{2} \theta_{j k}\right)^{2} r_{j k}^{-6}
\end{aligned}
$$

where $N$ is the number of interacting spins and $r$ the distance between the $j$ and $k$ spins. In powdered samples, which are what we have studied, the average over all orientations of the crystallites lead to the reduced formula (see [9]) :

$$
M_{2}=(3 / 5) \gamma^{4} \hbar^{2} I(I+1) N^{-1} \sum_{j>k} r_{j k}^{-6} .
$$

The overall second moment, $M_{2}$, can be separated into two parts [9], the intra- and inter-molecular contributions :

$$
M_{2}=M_{2, \text { intra }}+M_{2, \text { inter }} .
$$

Both parts can be reduced by fast molecular motions [8], so that we usually define $M_{2 \text {, intra/inter, rigid lattice }}$ when we have $\omega \tau \gg 1$ and $M_{2}$, intra/inter, averaged when $\omega \tau \ll 1$, where $\omega$ is the value of the local dipolar field expressed in frequency units and $\tau$ is the motions correlation time. We then have [9] :

$$
M_{2, \text { intra, rigid }}=7.16 \times 10^{-58} N^{-1} \sum_{j>k} r_{j k}^{-6}\left(\mathrm{G}^{2}\right) .
$$

For the thiophene molecule, we found $1.90 \mathrm{G}^{2}$.

For obvious geometrical reasons, the thiophene molecule can be thought of as experiencing in-plane reorientation. In this case, $M_{2 \text {, intra }}$ is reduced to a quarter of the rigid lattice value [8], i.e. $0.48 \mathrm{G}^{2}$. For isotropic motion this value would be averaged to zero.

The intermolecular part of the second moment closely depends on the structure of the studied phase. We have used structural models of André et al. [2, 4] for these calculations.

3.2.1 Phase I intermolecular second moment calculations. - For the sake of clarity, let us summarize the X-ray diffraction data analysis made by André et al. [2]. In order to build a structural model, one has to enumerate all the positions that one molecule can occupy at a crystal site, taking into account the molecular geometry and the crystal site symmetry. For thiophene phase I of space group Cmca, each molecule is located at a site of symmetry $2 / \mathrm{m}$ corresponding to 4 positions occupied with equal probabilities. The pseudo-pentagonal molecular symmetry being thought to provide 5 distinct equiprobable orientations, the total number of accessible orientations for the molecule amounts to $4 \times 5=$ 20 (see Fig. 1). This model provided the best fit to the experimental data [2].

Given this model, a group of molecules can experience many different local configurations as a 
function of space and time, giving rise to possibly different values of the intermolecular second moment. We have used two methods for estimating average rigid lattice intermolecular second moments, thereafter called method 1 and method 2 . In both methods we have placed a first molecule at a crystal site we called "central site", and then progressively filled the neighbouring sites up to a cut-off distance $d_{\max }$. The proton-proton distances were then calculated and inserted into equation (2).

In method 1 , each neighbouring site was assumed to be simultaneously occupied by all the $n$ possible molecular orientations. Each site computed contribution was then divided by $n$. The calculation has been performed for each of the 5 orientations of the central molecule which are not linked together by crystal site symmetry operations. The results are shown in table II for different values of $d_{\max }$. The mean second moment rapidly converges to a value of $5.47 \mathrm{G}^{2}$. It can be seen that very different local values of $M_{2}$ are found depending on the orientation of the central molecule. This gave us the idea of developing another method which could provide informations on the extent of the local $M_{2}$ distribution.

In method 2, all the sites including the central one were filled by random draws between the 20 possible orientations. We thus constructed many possible "globules» and studied the mean second moment value as a function of $d_{\max }$ and the number of computed globules. This is shown in table III. The mean values compare quite well, after convergence

Table II. - Thiophene, phase I. Intermolecular second moment as a function of $d_{\max }$ calculated through method-1 (see text) for each of the 5 molecular orientations which are not linked together by crystal site symmetry operations.

\begin{tabular}{|c|c|c|c|c|c|}
\hline $\begin{array}{r}d_{\max }(\AA) \\
\text { number of } \\
\text { neighbouring }\end{array}$ & $\begin{array}{r}7.5 \\
\text { sites }\end{array}$ & $\begin{array}{c}9.75 \\
(38)\end{array}$ & $\begin{array}{c}15 \\
(130)\end{array}$ & $\begin{array}{c}20 \\
(298)\end{array}$ & $\begin{array}{c}30 \\
(978) \\
\text { centre site } \\
\text { molecule }\end{array}$ \\
\hline 1 & 4.53 & 4.64 & 4.69 & 4.70 & 4.70 \\
\hline 2 & 5.11 & 5.22 & 5.28 & 5.29 & 5.29 \\
\hline 3 & 7.32 & 7.44 & 7.49 & 7.50 & 7.51 \\
\hline 4 & 4.58 & 4.70 & 4.76 & 4.77 & 4.77 \\
\hline 5 & 4.91 & 5.02 & 5.08 & 5.09 & 5.09 \\
\hline mean value & 5.29 & 5.40 & 5.46 & 5.47 & 5.47 \\
\hline
\end{tabular}

Table III. - Thiophene, phase I. Intermolecular second moment as a function of $d_{\max }$ for different number of " globules », calculated through method-2 (see text).

\begin{tabular}{|c|c|c|c|c|}
\hline $\begin{array}{c}\text { number } \\
\text { of globules }\end{array}$ & 7.5 & 9.75 & 15 & 20 \\
\hline 1000 & 5.10 & 5.45 & 5.55 & 5.34 \\
\hline 2000 & 5.13 & 5.45 & 5.57 & 5.53 \\
\hline 3000 & 5.17 & 5.47 & 5.56 & 5.50 \\
\hline 4000 & 5.18 & 5.44 & 5.50 & 5.47 \\
\hline 5000 & 5.18 & 5.43 & 5.48 & 5.45 \\
\hline 6000 & 5.18 & 5.40 & 5.49 & 5.42 \\
\hline 7000 & 5.20 & 5.41 & 5.46 & 5.42 \\
\hline 8000 & 5.20 & 5.41 & 5.47 & 5.43 \\
\hline 9000 & 5.23 & 5.40 & 5.47 & 5.43 \\
\hline 10000 & 5.25 & 5.41 & 5.45 & 5.44 \\
\hline
\end{tabular}

is obtained, with the values of table II, for identical cut-off distances. This is not surprising since method 2 consists in drawing randomly between all the configurations taken into account in method 1. The distribution of local second moments is shown in figure 6 for a 10000 globules draw. The shape of the distribution is complex and rather surprising. $95 \%$ of the values lie between 1 to $10 \mathrm{G}^{2}$, which is a much

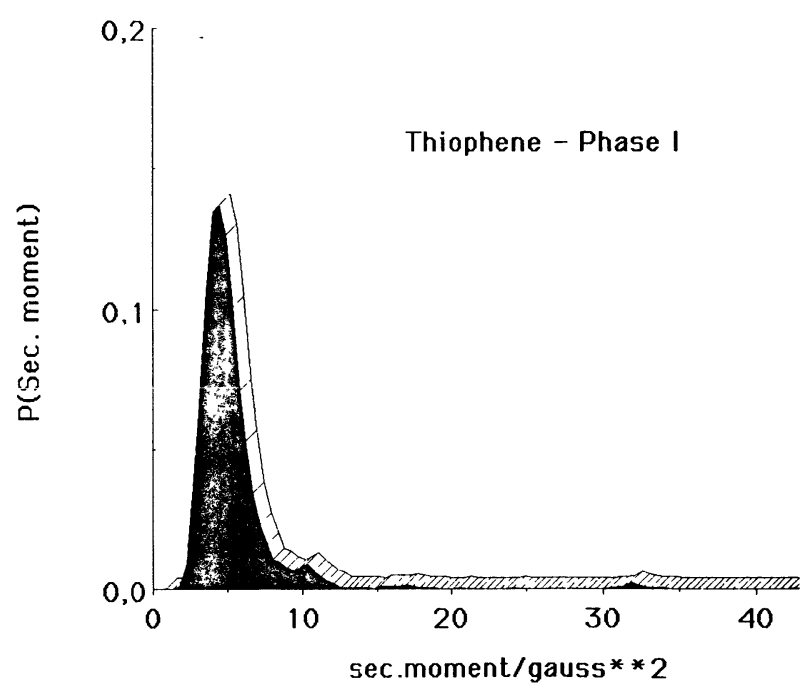

Fig. 6. - Thiophene, phase I : distribution of local second moment for a 10000 globules draw. 
wider distribution than expected, and moreover the remaining $5 \%$ are spread into two small components peaking at 17 and $31 \mathrm{G}^{2}$ ! This latter result indicates that some local configurations are not physically feasible because of steric hindrance. The second moment calculation acts as an amplifier for detecting local steric hindrance since the atom-atom distances appear to the power of six in the equations. If we retain only the configurations corresponding to intermolecular rigid lattice second moments $<10 \mathrm{G}^{2}$, the mean value drops from 5.42 to $4.90 \mathrm{G}^{2}$.

Experimentally, phase I is not observed in the rigid lattice situation. If we assume an in-plane molecular reorientation, the intermolecular $M_{2}$ should be reduced by a factor close to 8 , just like in solid benzene [8]. This would lead to a value of

$$
M_{2, \text { inter, in-plane }}=0.61 \text { to } 0.68 \mathrm{G}^{2}
$$

and to a total $M_{2}$ value of 1.09 to $1.16 \mathrm{G}^{2}$ which compares quite well with the observed $1.2 \pm 0.1 \mathrm{G}^{2}$. Besides obvious geometrical reasons, the in-plane motion assumption is more realistic than, for instance, an isotropic reorientation where $M_{2 \text {, intra }}$ would be averaged to zero and $M_{2 \text {, inter }}$ (calculated through the method which consists in placing all the nuclear spins at the centre of mass of the molecule) would be $0.73 \mathrm{G}^{2}$.

3.2.2 Phase III intermolecular second moment calculations. - In both phase sequences, the experimental second moment is slowly raising as temperature decreases, once the intense II to III transition has occurred (see Fig. 5). At low temperature $M_{2}$ is expected to finally reach the rigid lattice value where no molecular motion is faster than $10 \mathrm{kHz}$. In order to find whether this value has been reached at $110 \mathrm{~K}$, we should calculate the rigid lattice $M_{2}$ for phases $\mathrm{IV}$ and $\mathrm{II}_{2}$ (so far we have only obtained this value for phase I : $7.0 \pm 0.2 \mathrm{G}^{2}$ ). In the metastable sequence, no sharp phase transition occurs down to the lowest temperature [1]. Since all the structures are quite similar to each other [4], the rigid lattice $M_{2}$ is expected to increase by only a small amount, as temperature lowers, due to the slow increase of compactness. The phase I value can then be taken as the rigid lattice $M_{2}$ for the whole metastable sequence. In the stable sequence, the value could differ from that of phase I because of the structural change at the II to III transition. Unfortunately, crystallographic data are only available in phase III. We shall then assume that the calculated value is a lower bound for the low temperature stable phases.

We have calculated the phase III intermolecular $M_{2}$ contribution as we did above for phase I. The space group is here Pnma and the site symmetry is $m$. This leads to $2 \times 5=10$ distinct possible molecular orientations. Method 1 described above leads to essentially similar results as for phase I with a mean

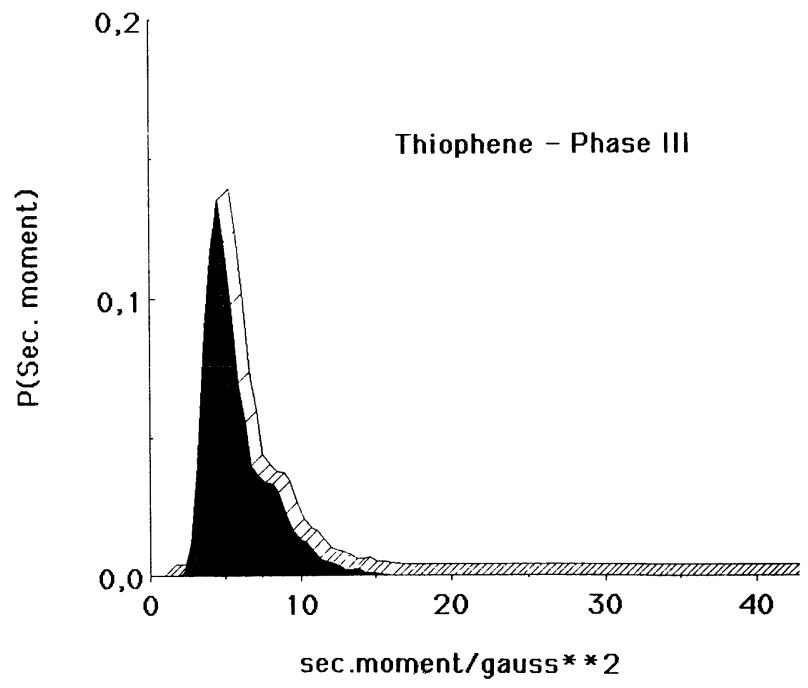

Fig. 7. - Thiophene, phase III : distribution of local second moments for a 10000 globules draw.

second moment value of $5.75 \mathrm{G}^{2}$. The results of method 2 calculations is shown in figure 7. The distribution of the local second moments is still wide but no sign of very large $\left(>15 \mathrm{G}^{2}\right)$ values appear in this phase. This might be due to the fact that potential wells have not been as extensively refined as in phase I (see [2]). The total rigid lattice $M_{2}$ amounts to $7.5 \pm 0.2 \mathrm{G}^{2}$, not so much different from the phase I value, keeping in mind that the data have not been recorded at the same pressure for both phases! We can then safely assume that the rigid lattice $M_{2}$ is of the order of 7 to $7.5 \mathrm{G}^{2}$ down to the lowest temperatures in both phase sequences.

3.2.3 Summary. - The experimental phase I second moment is approximately constant and its value of $1.2 \pm 0.1 \mathrm{G}^{2}$ is well understood in terms of fast inplane molecular reorientations. For all the other stable or metastable phases, $M_{2}$ is larger than $1.2 \mathrm{G}^{2}$ and smaller than the rigid lattice value of 7 to $7.5 \mathrm{G}^{2}$. This means that in the whole experimental temperature range, and presumably at even lower temperatures, the frequency of the molecular motion is of the order of magnitude of the local dipolar field (expressed in frequency units i.e. roughly $10 \mathrm{kHz}$ ). Taken for granted that the phase transformations do not change markedly the dynamic behaviour (at least in the metastable sequence where no orderdisorder transformation occurs), this result is still surprising because of the unusually wide range of temperature where $M_{2}$ increases. For instance, in the case of benzene, this range amounts to $25 \mathrm{~K}$ [9], instead of more than $100 \mathrm{~K}$ here.

\section{Discussion.}

Since the works of Van Vleck [7] and Andrew and Eades [9], calculating theoretical second moments is 
often considered as a straighforward task. However, most of the examples published in the literature concern materials with either rather simple crystalline structures or non-existent structural data. In this latter case, second moment values are often just guessed [6]. The thiophene example shows that this old fashioned kind of work can still provide its part of unexpected results.

Obviously thiophene molecules hardly stick together. Some recent work [22] shows that the steric incompatibilities are mostly concentrated in certain directions of space. Is this in any way related to the incommensurability and/or to the low temperature glass transition phenomenon? This will be the subject of a future work.

On the other hand, the extremely wide range of temperature where the second moment lies in-between the rigid lattice and the fast in-plane reorientation limits can be understood in terms of a distribution of correlation times [11], or, in a less phenomenological way, of an « unequal potential wells " structure of the molecular reorientation $[12,13]$. This follows immediately from symmetry considerations of the thiophene molecule and inplane reorientation. In the well known paper by Andrew and Eades [9], this fact appears quite clearly when comparing benzene and deutero-benzene behaviours. Moreover, we have measured the proton longitudinal relaxation times at $80 \mathrm{MHz}$ (Fig. 8) and observed a non-standard behaviour. The different slopes at the cold and the hot sides of the minima indicates a non-Lorentzian motion's autocorrelation function. This again can be interpreted in terms of distributions of correlation times [14, 15].

Several experimental results [16-18] as well as 'a recent model [19] have led to the idea that molecular asymmetry is closely related to the possibility of obtaining a glassy crystal. In thiophene, the dynamic

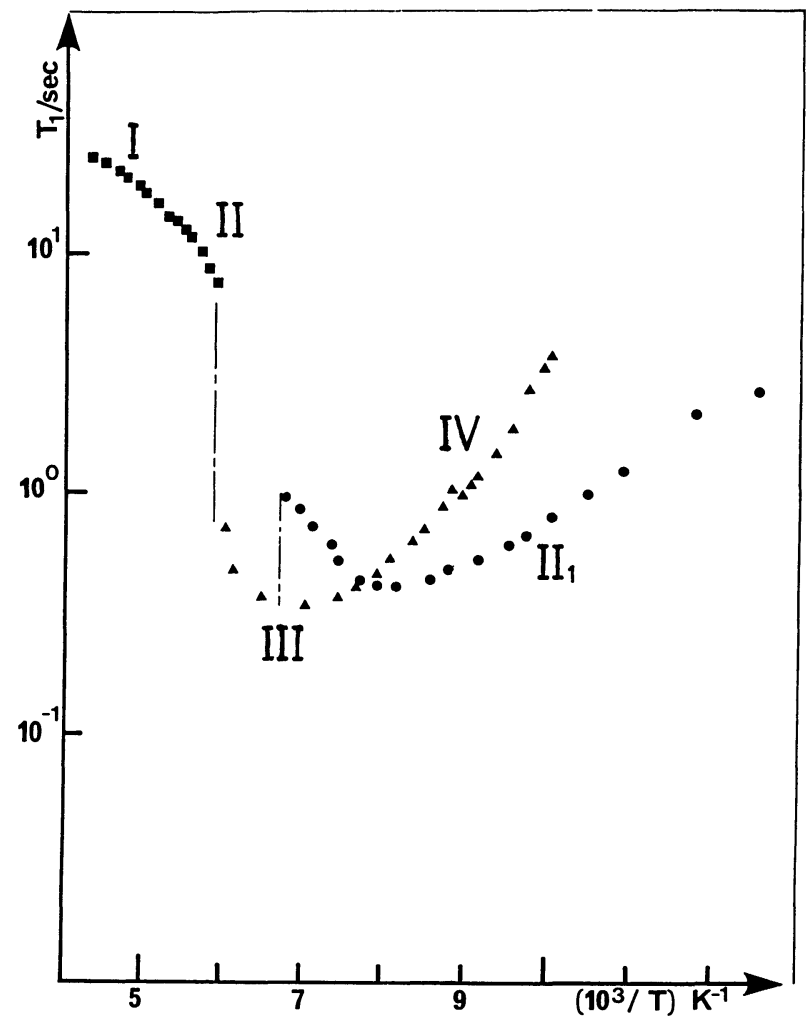

Fig. 8. - Thiophene : spin lattice relaxation times as a function of temperature at $80 \mathrm{MHz}$ : $\square$ plastic phase I ; $\Delta$ stable sequence ; $\bullet$ metastable sequence.

in-plane orientational disorder « survives » through several successive phase transitions. The progressive slowing-down of this motion is presumably correlated with the glass transitions observed at very low temperatures in both phase sequences. This point is fully developped in a forthcoming article [20].
[1] a) André, D., Dworkin, A., Figuière, P., Fuchs, A. H. and Szwarc, H., J. Phys. Chem. Solids 46 (1985) 505.

b) Figuière, P., Szwarc, H., Oguni, M. and SugA, S., J. Chem. Thermodyn. 17 (1985) 949.

[2] André, D., Figuière, P., Fourme, R., GhelfenSTEIN, M., Labarre, D. and SzWarc, H., $J$. Phys. Chem. Solids 45 (1984) 299.

[3] André, D., Dworkin, A., Figuière, P., Fuchs, A. H. and Szwarc, H., C. R. Hebd. Séan. Acad. Sci. Paris Ser. II 295 (1982) 145.

[4] ANdré, D. and SzwARC, H., J. Phys. France 47 (1986) 61.

[5] Sanford, W. E. and Boyd, R. K., Can. J. Chem. 54 (1976) 2773.
[6] Fried, F., Thèse, Nice A.O. C.N.R.S. 9740 (1974).

[7] Van Vleck, J. H., Phys. Rev. 74 (1948) 1168.

[8] Abragam, A., Les principes du Magnétisme Nucléaire (INSTN, PUF, Paris) 1961, 449.

A direct calculation of the intermolecular second moment reduction by an in-plane motion is theoretically possible with the method developed by Rigny [18]. In practice the complexity of the phase I structure makes it difficult to achieve such a calculation. Beside, benzene and thiophene have closely similar structures. They form a continuous series of solid solutions over the entire range of composition [21].

[9] Andrew, E. R. and Eades, R. G., Proc. R. Soc. A 216 (1953) 398. 
[10] Bartis, F. J., Nature 268 (1977) 427 ; Philos. Mag. A 52 (1985) L33.

[11] Connor, T. H., Trans. Farad. Soc. 60 (1964) 1574.

[12] Anderson, J. E., J. Magn. Res. 11 (1973) 398.

[13] Polack, M. and Ailion, D. C., J. Chem. Phys. 67 (1977) 3029.

[14] Bloembergen, N., Purcell, E. M. and Pound, R. V., Phys. Rev. 73 (1948) 679.

[15] Fuchs, A. H., Virlet, J., ANDrÉ, D. and Szwarc, H., J. Chim. Phys. 82 (1985) 293.
[16] Fuchs, A. H., Thèse de doctorat, AO 2802, Orsay (1983).

[17] Bessada, C., Doctorat en Sciences, Orsay (1987).

[18] Rigny, P., Physica 59 (1972) 707.

[19] Szwarc, H., Thermochim. Acta 109 (1986) 91.

[20] Bessada, C., Fuchs, A. H. and Gallier, J., to be published.

[21] Fawcett, F. S. and Rasmussen, J., J. Am. Chem. Soc. 67 (1945) 1705.

[22] ANDRÉ, D. and SZWARC, H., to be published. 